\title{
Effectiveness of acupuncture in the management of restless leg syndrome: a systematic review and meta-analysis
}

\author{
Cheng Huang ${ }^{1 \#}$, Jia-Fa Tang ${ }^{2 \#}$, Wei Sun ${ }^{3}$, Li-Zhe Wang ${ }^{4}$, Yi-Si Jin ${ }^{2}$ \\ ${ }^{1}$ Department of Integrated Traditional Chinese and Western Medicine, Daqing Oilfield General Hospital, Daqing, China; ${ }^{2}$ Department of \\ Rehabilitation, Daqing City People's Hospital, Daqing, China; ${ }^{3}$ Department of Traditional Chinese Medicine, Daqing Oilfield General Hospital, \\ Daqing, China; ${ }^{4}$ Department of Integrated Traditional Chinese and Western Medicine, Heilongjiang Provincial Hospital, Harbin, China \\ Contributions: (I) Conception and design: All authors; (II) Administrative support: None; (III) Provision of study materials or patients: None; (IV) \\ Collection and assembly of data: None; (V) Data analysis and interpretation: LZ Wang, W Sun; (VI) Manuscript writing: All authors; (VII) Final \\ approval of manuscript: All authors. \\ "The authors contributed equally to this work. \\ Correspondence to: Dr. Yi-Si Jin. Department of Rehabilitation, Daqing City People’s Hospital, 213 Jianshe Road, Daqing 163316, China. \\ Email: dr_jinyisi2468@163.com.
}

Background Acupuncture is an important component of traditional Chinese medicine which is used to treat an array of health conditions. This study evaluates the effectiveness of acupuncture in the management of restless leg syndrome (RLS).

Methods: A comprehensive literature survey was conducted in electronic databases to identify studies evaluating the effectiveness of acupuncture in the treatment of RLS. Cure, marked effect, effective, ineffective, and total effective rates of individual studies were pooled to achieve their respective overall estimates and a meta-analysis of mean change from baseline in International Restless Legs Syndrome Rating Scale (IRLSRS) was performed.

Results: A total of 18 studies were included in this meta-analysis, among which 640 RLS patients were treated with acupuncture alone or combined with other therapies (acupuncture group) and 447 RLS patients were treated with non-acupuncture therapies (control group). Cure, marked effect, and effective rates were $47.8 \%$ [ $95 \%$ confidence interval (CI): $38.3 \%$ to $57.3 \%$ ], $27.4 \%$ (95\% CI: $20.3 \%$ to $34.8 \%$ ), and $24.2 \%$ (95\% CI: $16.9 \%$ to $31.5 \%$ ) in patients treated with acupuncture either alone or combination with other treatments, and $21.7 \%$ ( $95 \%$ CI: $20.7 \%$ to $22.7 \%$ ), $28.0 \%$ (95\% CI: $20.0 \%$ to $32.9 \%$ ), and $22.3 \%$ (95\% CI: $17.0 \%$ to $27.6 \%)$ in patients treated with non-acupuncture therapies, respectively. The ineffective rate was $4.7 \%(95 \%$ CI: $4.3 \%$ to $5.0 \%$ ) in the acupuncture group and $32.9 \%$ (95\% CI: $22.2 \%$ to $43.7 \%$ ) in non-acupuncture groups. IRLSRS scores improved significantly after acupuncture treatment [mean change from baseline -9.45 (95\% CI: -18.42 to -0.49$)$; $\mathrm{P}=0.04]$.

Discussion: Although the overall quality of the included studies was low, the results of this meta-analysis suggested that acupuncture is an effective treatment option for RLS. Well-designed randomized controlled trials especially those involving a sham-acupuncture arm are needed to confirm these outcomes.

Keywords: Restless leg syndrome (RLS); acupuncture; moxibustion; cure; effect

Submitted Jul 27, 2021. Accepted for publication Oct 09, 2021.

doi: 10.21037/apm-21-2309

View this article at: https://dx.doi.org/10.21037/apm-21-2309 


\section{Introduction}

Restless leg syndrome (RLS) is a "movement-responsive nocturnal focal akathisia with dysesthesia" (1) characterized by an urge to fold and unfold the legs rapidly accompanied by an unpleasant sensation that interferes with sleep. These symptoms, which most often arise at night, are worse at rest and are relieved upon moving legs or walking $(2,3)$. There are 2 forms of RLS: primary RLS has a strong hereditary background and onsets early in life to peak at an age of 20 years. Up to $40-60 \%$ of individuals with RLS have a family history of RLS. Secondary RLS is an adult condition and appears later in life. It is characterized by sporadic symptoms with slower regression which may also be associated with peripheral neuropathy (3).

The condition of RLS is under-diagnosed. The prevalence of RLS is reported to be between less than $1 \%$ to $15 \%$ in various parts of the world $(11.4 \%$ for Swedish women and $5.8 \%$ for Swedish men, $10.8 \%$ for French women and $5.8 \%$ for French men, $9.8 \%$ for German elderly 65-83 years old, $10 \%$ in Canada, and $1 \%$ in Singapore) (4). RLS has been found to be associated with risk of cardiovascular disease (CVD) even after controlling for age, gender, race, body mass index, systolic blood pressure, antihypertensive drug use, diabetes, cholesterol, smoking, and apnea-hypoapnea index $(5,6)$. Insomnia and daytime distress have also been found to be significantly associated with RLS (7). Age, pregnancy, lower socioeconomic status, low iron levels, poor health, poor mental health, Parkinson's disease, and end-stage renal disease have been identified as the risk factors for RLS (3).

Dopaminergic drugs such as levodopa are the firstline treatment for RLS (8). The analogues of gammaaminobutyric acid such as gabapentin have been shown to be safe and efficacious in treating RLS of hemodialysis patients $(9,10)$. Opioids, benzodiazepines, 4-phenylpiperidine analogues, and alpha-2 adrenergic receptor agonists are also used for RLS treatment (11). Repetitive transcranial magnetic stimulation, pneumatic compression devices, counter-strain manipulation, infrared therapy, vibration pads, yoga, cryotherapy, and acupuncture are among the notable non-pharmacological treatments of RLS.

Acupuncture is an important component of traditional Chinese medicine (TCM) which is used to treat an array of health conditions. Insertion of acupuncture needles in various acupoints targeting energy channels is postulated to restore a balance that maintains health (12). Several studies have reported the outcomes of acupuncture in the management of RLS either as sole treatment or in combination with other related therapies, but the designs and the outcomes vary across these studies which necessitates a systematic review of this topic. The aim of the present study was to evaluate the effectiveness of acupuncture for the treatment of RLS by reviewing the outcomes of relevant studies in literature. We present the following article in accordance with the PRISMA reporting checklist (available at https://dx.doi. org/10.21037/apm-21-2309).

\section{Methods}

\section{Eligibility criteria}

The inclusion criteria of the present study were: a study (I) evaluated the effectiveness of acupuncture either alone or with other related therapies for the management of RLS; (II) reported quantitative outcomes depicting the efficacy; and (III) reported the outcome measure/s which could depict the effectiveness in alleviating the symptoms of RLS such as sleep quality, pain, activity, and so on. The exclusion criteria were: other TCM methods were utilized without acupuncture; reported the outcomes without differentiating acupuncture from other therapies; or did not report quantitative information.

\section{Literature search}

For literature search, electronic databases consulted were: China National Knowledge Infrastructure (CNKI), Google Scholar, PubMed, and Wangfang. The important medical subject headings and keywords used as different phrases were: restless leg syndrome, RLS, acupuncture, acupoints, electro-acupuncture, hydro-acupuncture, tuina, massage, moxibustion, acuinjection, warm needle, stone scraping, traction, plum-blossom, and cupping. The search was delimited to original research papers published in English or Chinese language before October 2020.

\section{Data and analyses}

Important data pertaining to the demographic and clinical characteristics of patients, treatments, outcome measures, and outcomes were extracted from research articles by 2 authors independently. Inter-rater reliability was high (Cohen kappa $=0.94)$. The primary endpoint for the 


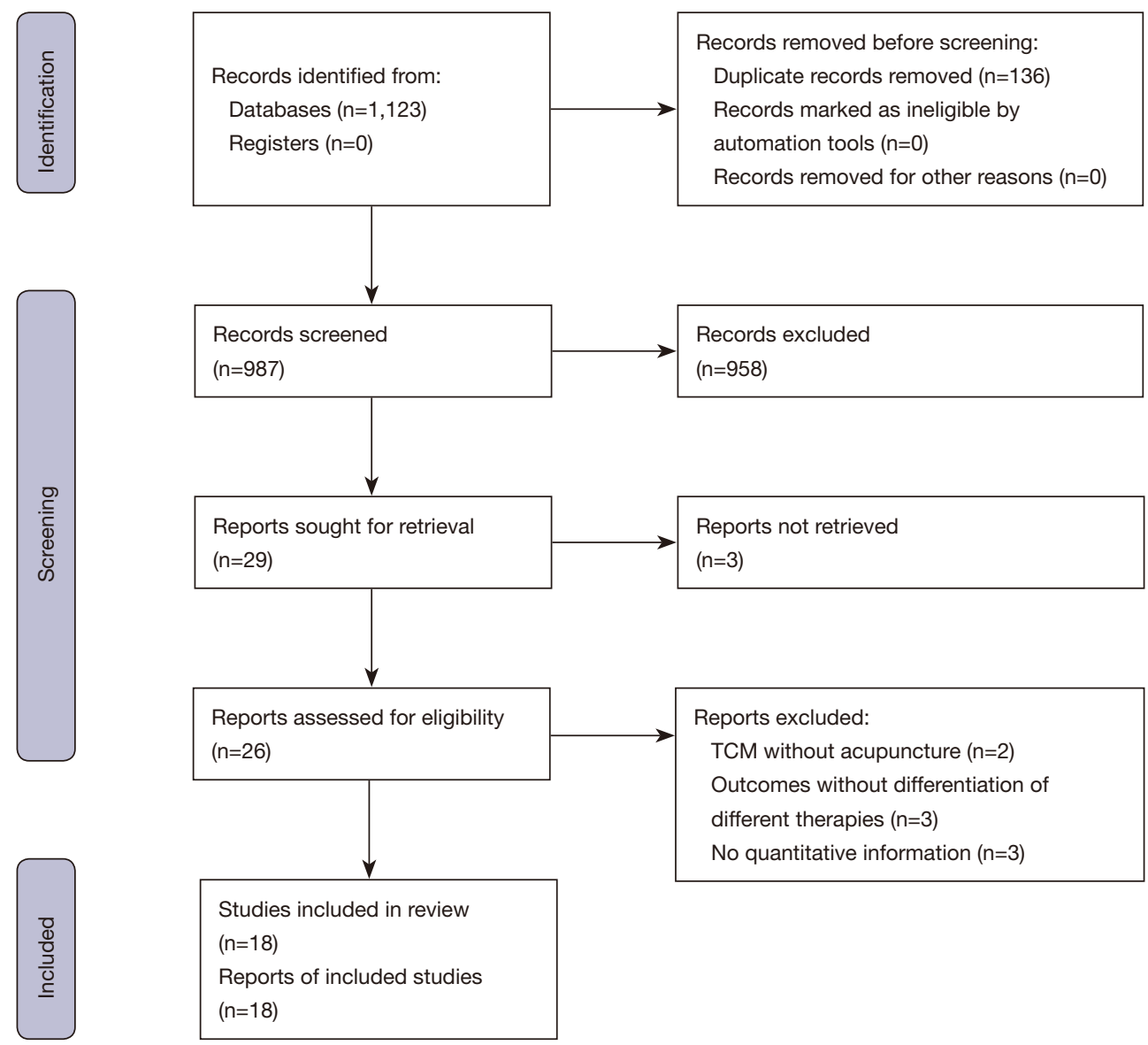

Figure 1 Flowchart of the study screening and selection process. TCM, traditional Chinese medicine.

present study was 'total effective rate' defined as the sum of 'cure rate', 'marked effect rate', and 'effective rate' of the treatment, where 'cure' was the disappearance of the symptoms of RLS with sufficient sleep ( $\geq 6 \mathrm{~h}$ ); 'marked effect' was remission of the symptoms of RLS with almost normal sleep (5-6 h); 'effective rate' was relieving of the symptoms of RLS with alleviation of insomnia (4-5 h sleep); and 'ineffective rate' meant no remission of the symptoms (13). Additional endpoints were the changes in the International Restless Legs Syndrome Rating Scale score, Epworth Sleep Scale score, and the Pittsburgh Sleep Quality Index score.

For achieving the pooled effect size of the percent effectiveness rates, random effects meta-analyses were performed with Stata software (version 12; Stata Corp.; College Station, TX, USA) using DerSimonian-Liard method where the standard errors of the effectiveness rates were derived from sample sizes.

\section{Statistical analysis}

For the estimation of the change in the International Restless Legs Syndrome Rating Scale scores, a metaanalysis of mean differences was performed with Cochrane Review Manager software (version 5.3) using randomeffects inverse-variance method.

Quality assessment of the included studies was performed with Newcastle-Ottawa Scale for the assessment of quality of observational cohort studies. Between-study inconsistency in outcomes (statistical heterogeneity) was estimated with $\mathrm{I}^{2}$ index and sensitivity analyses were performed. Begg's test was performed to assess publication bias.

\section{Results}

A total of 18 studies (13-30) were included in this metaanalytical review (Figure 1). In these studies, 640 patients 
with RLS were treated with acupuncture either alone or in combination with any other therapy and 447 patients were treated with non-acupuncture control therapies. A total of 10 studies performed acupuncture alone and rest of the studies performed acupuncture in combination with other therapies including moxibustion $(n=3)$, cupping $(\mathrm{n}=1)$, injection $(\mathrm{n}=1)$, TCM herbal fumigation $(\mathrm{n}=1)$, gabapentin $(n=1)$, and levodopa $(n=1)$. Control treatments included levodopa with or without benserazide $(n=5)$, non-acupuncture TCM $(\mathrm{n}=1)$, alprazolam/oryzanol $(\mathrm{n}=1)$, gabapentin ( $\mathrm{n}=1)$, and vitamin $\mathrm{B}_{1 / 12}$-Danshen $(\mathrm{n}=1)$. Quality of the included study was low to moderate in general (Table 1).

The total effective rate was $90.5 \%$ [ $95 \%$ confidence interval (CI): $86.2 \%$ to $94.9 \%$ ] in patients treated with acupuncture either alone or in combination with other related treatments and $65.8 \%$ (95\% CI: $52.1 \%$ to $79.5 \%$ ) in patients treated with non-acupuncture therapies (Figure 2). Cure, marked effect, and effective rates were $47.8 \%$ (95\% CI: $38.3 \%$ to $57.3 \%$ ), $27.4 \%$ (95\% CI: $20.3 \%$ to $34.8 \%$ ), and $24.2 \%$ (95\% CI: $16.9 \%$ to $31.5 \%$ ) in patients treated with acupuncture either alone or in combination with other treatments and $21.7 \%$ (95\% CI: $20.7 \%$ to $22.7 \%$ ), $28.0 \%$ (95\% CI: $20.0 \%$ to $32.9 \%$ ), and $22.3 \%$ (95\% CI: $17.0 \%$ to $27.6 \%)$ in patients treated with non-acupuncture therapies, respectively. The ineffective rate was $4.7 \%$ (95\% CI: $4.3 \%$ to $5.0 \%$ ) in the acupuncture group and $32.9 \%$ (95\% CI: $22.2 \%$ to $43.7 \%$ ) in non-acupuncture groups.

There was no difference in the total effective rate between acupuncture alone [91.6\% (95\% CI: 90.6\% to $92.6 \%)$ ] and acupuncture in combination with other therapies [92.2\% (95\% CI: 90.9\% to $93.5 \%)$ ]. Cure rate was higher in patients treated with acupuncture alone [51.1\% (95\% CI: $44.4 \%$ to $57.8 \%$ )] than in those treated with acupuncture in combination with other related therapies [44.1\% (95\% CI: $25.9 \%$ to $62.2 \%$ ); Figure 3], but marked effect rate was lower in patients treated with acupuncture alone [16.1\% (95\% CI: $11.0 \%$ to $21.2 \%$ )] than in those treated with acupuncture in combination with other related therapies [38.7\% (95\% CI: $24.0 \%$ to $53.4 \%$ ); Figure 4$]$. There was not much difference in the effective rate $[24.8 \%$ (95\% CI: 16.5 to 33.1 ) of acupuncture alone vs. $23.5 \%$ (95\% CI: $12.5 \%$ to $34.6 \%$ ) combination] but ineffective rate was slightly higher in the acupuncture group [9.22\% (95\% CI: $5.36 \%$ to $13.1 \%$ )] than in acupuncture in combination with other therapies [2.48\% (95\% CI: $2.12 \%$ to $2.84 \%$ )].

A meta-analysis of 2 studies showed significant improvement in International Restless Legs Syndrome
Rating Scale scores after acupuncture treatment [mean change from baseline -9.45 (95\% CI: -18.42 to -0.49 ); $\mathrm{P}=0.04$; Figure 5].

\section{Discussion}

In this meta-analysis, we have found that acupuncture, especially in combination with other related therapies, was more effective than non-acupuncture therapies for RLS. However, some methodological issues kept this evidence at a low level. Only a few studies reported information about the number of acupuncture sessions performed; follow-up ranged from 1 month to 2 years; and majority of the included studies reported effectiveness rates without symptomatic improvement indices. Thus, well-designed randomized controlled trials are needed to confirm the findings of the present study.

Acupuncture is one of the important components of TCM that is recognized as an efficacious and safe treatment option for many health conditions including neurological and psychiatric diseases. Acupoints such as Shenshu (BL23), Xuehai (SP10), Chenshan (BL57), Zusanli (ST36), Sanyinjiao (SP6), and Taixi (KD3) are commonly involved in the treatment of RLS (31). As far as the mechanism of action is concerned, it is suggested that acupuncture may target the autonomic nervous system to manifest its effects. Acupuncture at Jianshi-Neiguan acupoints (PC5 to PC6 below median nerve) inhibits the sympathetic nerve and slows down excitatory cardiovascular reflexes via the opiate pathways which may trigger the inflammatory reflex and immune system (21). Moreover, acupuncture is reported to reduce oxidative stress in brain parts and promotes neuroprotection by increasing levels of BDNF, GDNF, and cyclophilin (32). Besides reducing oxidative stress, acupuncture treatment is also found to exhibit antiapoptotic and anti-inflammatory effects (33).

Acupuncturists emphasize that Yang deficiency in the legs causes symptoms like sensation of cold or fatigue in lower extremity whereas Yang stagnation which may represent disturbance of Shao Yang affects muscle tension. It is suggested that for improving Yang deficiency in the legs, needling in Tianshu (ST25) and Juxu Shanglian (ST37) acupoints can be useful whereas for the improvement in Yang stagnation, the acupoints Zhongdu (GB32) and Xiaoluo (TB12) are needed to be activated. Qi stagnation may also cause restlessness in the legs which can be treated by needling Dadun (LR1) and Yanglingquan (GB34). Ying deficiency and Yang stagnation leads to symptoms 


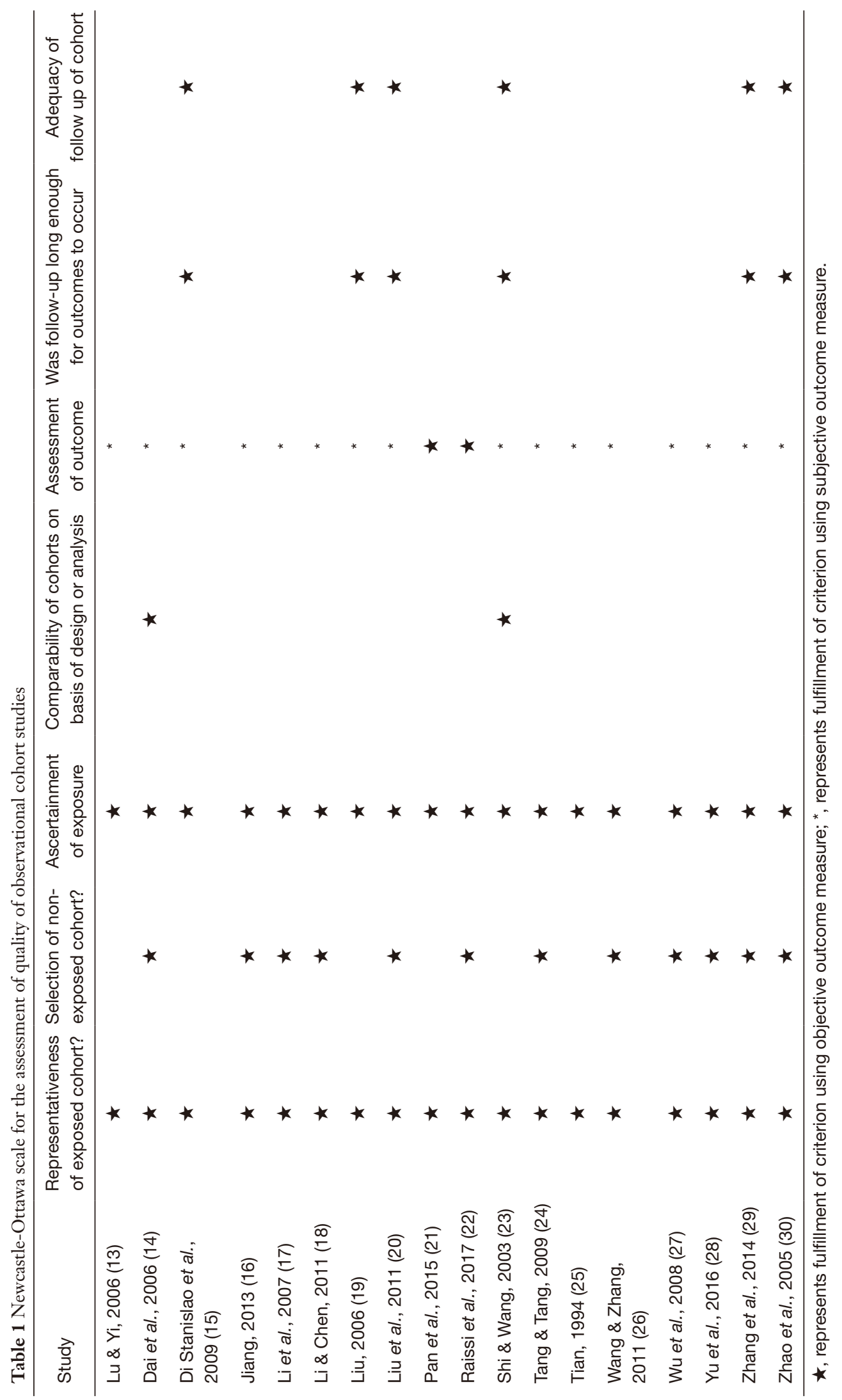




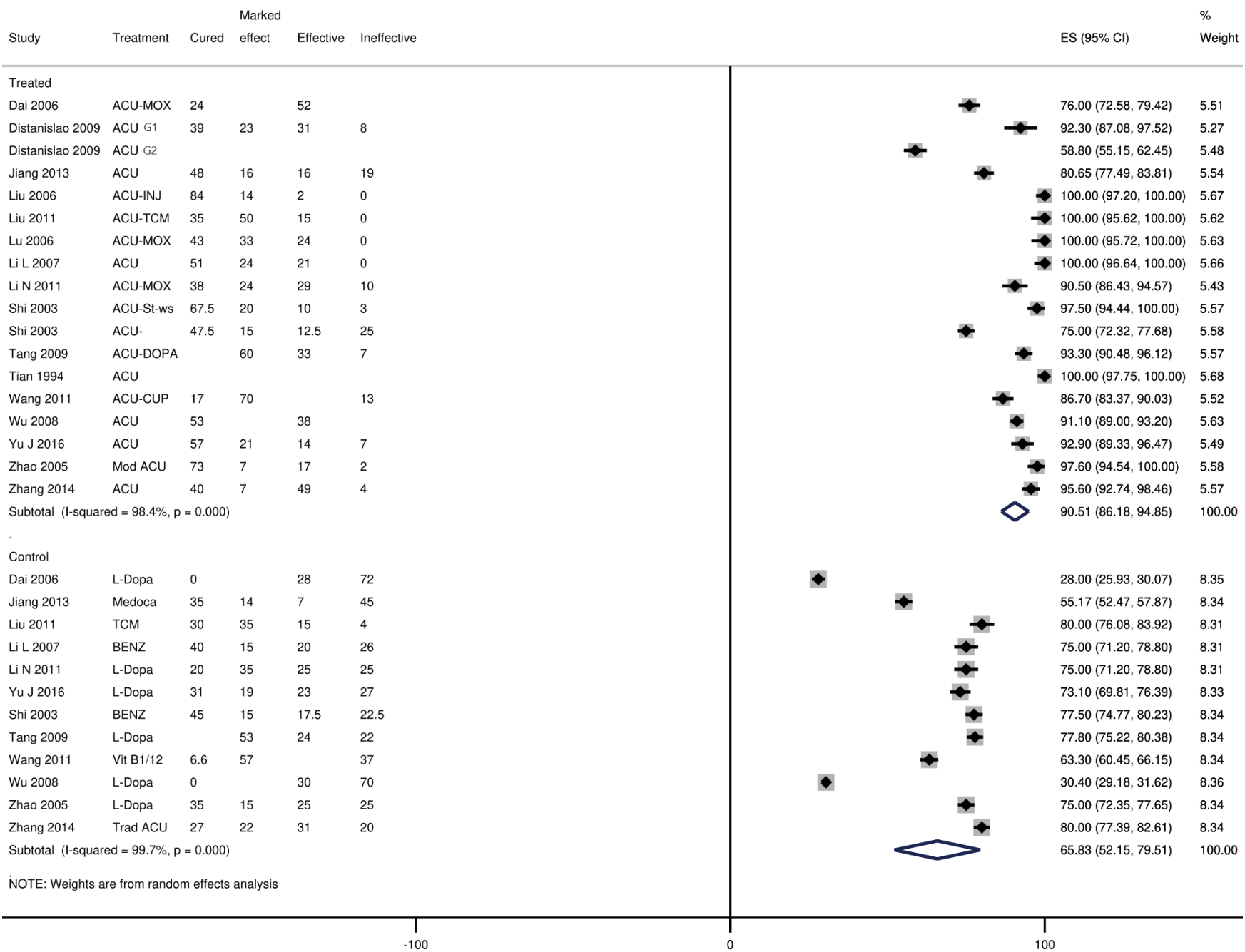

Figure 2 Forest graph showing the total effective rates of treated and control groups. Columns after treatment are the percent values of cure, marked effect, effective, and ineffective rates. ACU, acupuncture; BENZ, bezodiazepine; DOPA-based therapy, dopamine, INJ, injection; L-DOPA, levodopamine, Mod, modern; MOX, moxibustion; St-ws, streaming and washing; TCM, traditional Chinese medicine; Trad, traditional; Vit, Vitamin.

like cramps, cold feet, insomnia, urinary inconsistency, muscle tension, and urge for motion. Acupoints useful to address such symptoms are Zhongdu GB32 and LR6 (15). According to Pan et al., liver yin and xue control lower limb activity at night and are also responsible for body and brain relaxation. When yin and xue become deficient, which may also be associated with qi imbalances, the conditions like RLS develop (21).

Among the included studies, Dai et al. (14) used Biguan (ST31) acupoint located on the line joining anterior superior iliac spine and the external edge of patella to dilate blood vessels and to activate qi which was substantiated by the moxibustion heat conducting down the leg. Liu (19) who treated 49 RLS patients found a total eradication of symptoms in 41 patients and relief was persistent by 6-months follow-up. Liu (19) used Yanglingquan (GB34), Jinggu (BL64), Chengshan (BL57), Chengjin (BL57), Shangju (SP5), Ganshu (BL18), Shenshu (BL23), Taixi (KI3), Xuehai (SP10) and Sanyinjiao (SP6) acupoints. Pan et al. (21) used Shenshu (BL23), Mingmen (DU4), Xuehai (SP10), Chenshan (BL57), Taichun (LR3), Zusanli (ST36), Sanyinjiao (SP6), and Taixi (ST36) acupoints to apply standard acupuncture and found it effective in improving symptoms and sleep quality. Raisi 


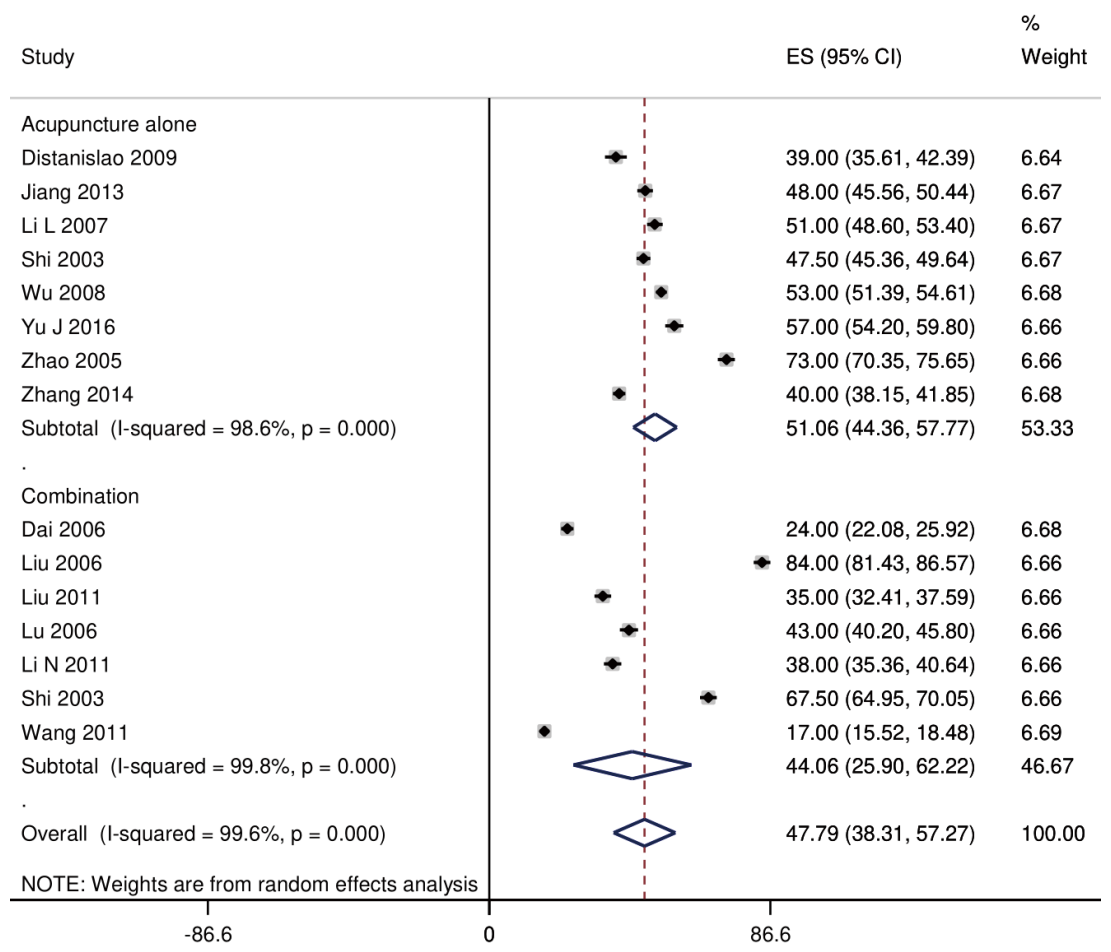

Figure 3 Forest graph showing the cure rates of patients treated with acupuncture alone and acupuncture in combination with other related therapies.

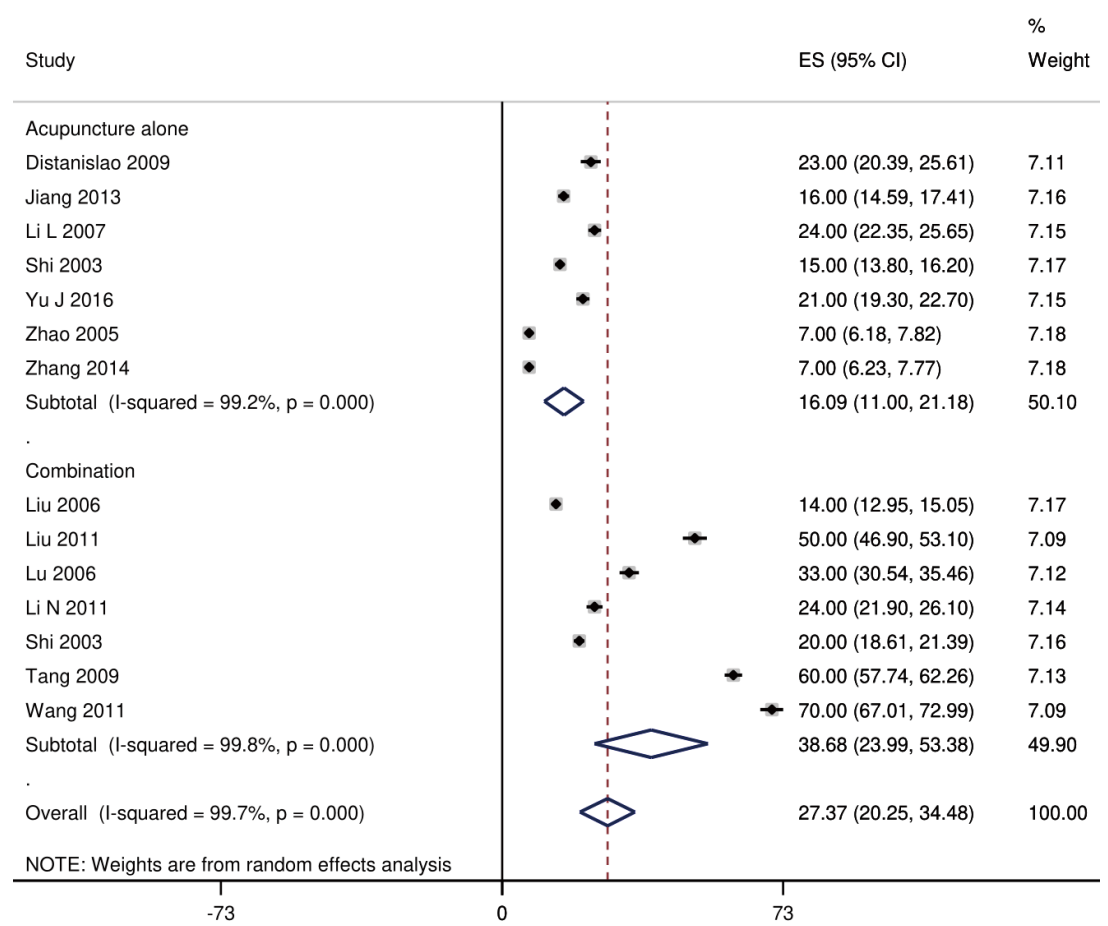

Figure 4 Forest graph showing the marked effect rates of patients treated with acupuncture alone and acupuncture in combination with other related therapies. 


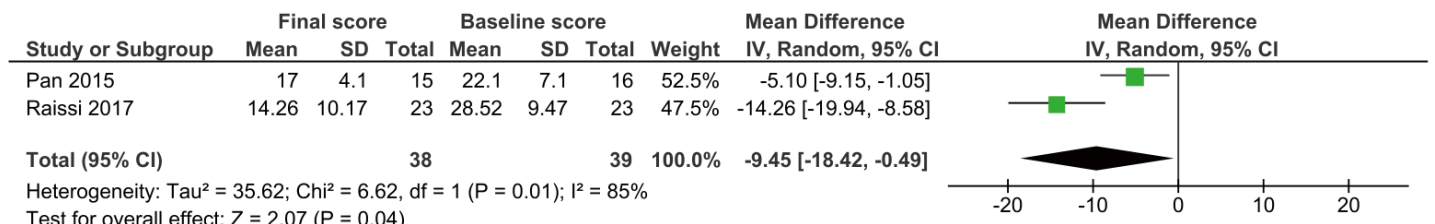

Figure 5 Forest graph showing the meta-analysis of mean change from baseline in International Restless Legs Syndrome Rating Scale score after acupuncture treatment.

et al. (22) who treated RLS patients with acupuncture in combination with gabapentin, used Shenshu (BL23), Ganshu (BL18), Chenshan (BL57), Jinggu (BL64), Zusanli (ST36), Liangqiu (ST34), Shangqiu (SP5), Xuehai (SP10), Shanyijiao (SP6), Taixi (KI3), Yanglingquan (GB34), Xuanzhong (GB39) acupoints and found that the combinational therapy had some additive effect in comparison with gabapentin monotherapy.

In the present study, a meta-analysis of 2 studies found a statistically significant improvement in International Restless Legs Syndrome Rating Scale scores 6-8 weeks after acupuncture treatment. Pan et al. (21) also found changes in the Epworth Sleep Scale score, and sleep mean activity to be significantly better in acupuncture group than in control group 6 weeks after treatment. Raissi et al. (22) found a significant difference in the Pittsburgh Sleep Quality Index score between acupuncture and control groups 8 weeks after treatment.

Some other studies have also reported that acupuncture is an effective treatment option for RLS. In a retrospective analysis of 19 patients, Cripps (34) found acupuncture efficacious in alleviating the symptoms of RLS. He used BL57 and LR3 acupoints bilaterally and stimulated these with electroacupuncture for 20-30 minutes. Greater improvement was noticed in patients who had not received previous dopaminergic therapy. In a single-blind, randomized controlled trial, 60 hemodialysis patients with RLS received 12 sessions of near infrared (940 nm) treatment to acupoints in the lower limb 3 times a week. In this study, clinically significant improvement was observed, but upon discontinuation the effect was diminished (35). Describing 2 cases with RLS who were treated with acupuncture, Fukutome (36) reported that $4 \mathrm{~W}$-acuinjection in Zulinqi (GB41), Kunlun (BL60), Zusanli (ST36), and Sanyinjiao (SP6) acupoints led to immediate relief in the form of a calm sensation and cessation of involuntary movements in legs.
A recent review found that besides acupuncture, other non-pharmacological treatments including repetitive transcranial stimulation, precise physical exercises, sequential pneumatic compression devices, use of counterstain techniques, and infrared exposure also remain effective in relieving RLS symptoms (37). Regardless of the treatment strategy, several factors can have impacts on the incidence, prevalence, and treatment of RLS. Among the socioeconomic factors, female gender, unemployment, retirement, less education, and low income are found to be associated with RLS (38). Depression is frequently associated with RLS. In a study of community-dwelling elderly, 1 in 8 individuals with RLS had depression whereas 1 in 25 individuals without RLS had depression. Men with RLS were more likely to take antidepressant medication (39). There is evidence to suggest that the use of antidepressant medication can aggravate RLS (40).

Acupuncture effects observed in trials are often suspected for the presence of placebo effect or expectation bias $(41,42)$. However, a recent randomized controlled trial found that raising expectations of the participants before acupuncture treatment had no effect on the outcomes, but the incidence of side effects was significantly more in the group that was intensely briefed about side effects possibilities (43). It is suggested that addition of a sham-acupuncture arm in acupuncture trials can yield a better clarity of the outcomes (44).

Among the notable limitations of the present study, less availability of non-Chinese literature is an important consideration. We observed high statistical heterogeneity in the pooled analysis which could be attributed to the use of many types of controls and many therapeutic combinations with acupuncture. To test sources of heterogeneity, we performed meta-regression analyses but neither study sample size nor follow-up duration, or year of study publication were significantly associated with total effective rate. Definitions of effectiveness rates were not given by 
most of the included studies and there may have been some between-studies deviation in measuring the actual effect of therapy.

\section{Conclusions}

According to this meta-analysis, acupuncture either alone or in combination with other related therapies is an effective treatment option for RLS. Cure rate significantly differed between acupuncture and non-acupuncture therapies (48\% vs. $22 \%$ ). Several limitations, including the variations in combinational and control treatments, methodological inconsistencies, variation in follow-up durations, and the lack of effect measurement standards kept the level of this evidence low. Therefore, randomized trials with better designs and suitable controls especially with a shamacupuncture arm are required to seek refined evidence.

\section{Acknowledgments}

Funding: None.

\section{Footnote}

Reporting Checklist: The authors have completed the PRISMA reporting checklist. Available at https://dx.doi. org/10.21037/apm-21-2309

Conflicts of Interest: All authors have completed the ICMJE uniform disclosure form (available at https://dx.doi. org/10.21037/apm-21-2309). The authors have no conflicts of interest to declare.

Ethical Statement: The authors are accountable for all aspects of the work in ensuring that questions related to the accuracy or integrity of any part of the work are appropriately investigated and resolved. All analyses were based on previous published studies, thus no ethical approval and patient consent to participate were required.

Open Access Statement: This is an Open Access article distributed in accordance with the Creative Commons Attribution-NonCommercial-NoDerivs 4.0 International License (CC BY-NC-ND 4.0), which permits the noncommercial replication and distribution of the article with the strict proviso that no changes or edits are made and the original work is properly cited (including links to both the formal publication through the relevant DOI and the license).
See: https://creativecommons.org/licenses/by-nc-nd/4.0/.

\section{References}

1. Benes H, Walters AS, Allen RP, et al. Definition of restless legs syndrome, how to diagnose it, and how to differentiate it from RLS mimics. Mov Disord 2007;22 Suppl 18:S401-8.

2. Allen RP, Picchietti D, Hening WA, et al. Restless legs syndrome: diagnostic criteria, special considerations, and epidemiology. A report from the restless legs syndrome diagnosis and epidemiology workshop at the National Institutes of Health. Sleep Med 2003;4:101-19.

3. Yeh P, Walters AS, Tsuang JW. Restless legs syndrome: a comprehensive overview on its epidemiology, risk factors, and treatment. Sleep Breath 2012;16:987-1007.

4. Tison F, Crochard A, Léger D, et al. Epidemiology of restless legs syndrome in French adults: a nationwide survey: the INSTANT Study. Neurology 2005;65:239-46.

5. Winkelman JW, Finn L, Young T. Prevalence and correlates of restless legs syndrome symptoms in the Wisconsin Sleep Cohort. Sleep Med 2006;7:545-52.

6. Winkelman JW, Shahar E, Sharief I, et al. Association of restless legs syndrome and cardiovascular disease in the Sleep Heart Health Study. Neurology 2008;70:35-42.

7. Broman JE, Mallon L, Hetta J. Restless legs syndrome and its relationship with insomnia symptoms and daytime distress: epidemiological survey in Sweden. Psychiatry Clin Neurosci 2008;62:472-5.

8. Scholz H, Trenkwalder C, Kohnen R, et al. Dopamine agonists for restless legs syndrome. Cochrane Database Syst Rev 2011;(3):CD006009.

9. Razazian N, Azimi H, Heidarnejadian J, et al. Gabapentin versus levodopa-c for the treatment of restless legs syndrome in hemodialysis patients: a randomized clinical trial. Saudi J Kidney Dis Transpl 2015;26:271-8.

10. Micozkadioglu H, Ozdemir FN, Kut A, et al. Gabapentin versus levodopa for the treatment of Restless Legs Syndrome in hemodialysis patients: an open-label study. Ren Fail 2004;26:393-7.

11. Yaltho TC, Ondo WG. The use of gabapentin enacarbil in the treatment of restless legs syndrome. Ther Adv Neurol Disord 2010;3:269-75.

12. Bega D, Malkani R. Alternative treatment of restless legs syndrome: an overview of the evidence for mind-body interventions, lifestyle interventions, and neutraceuticals. Sleep Med 2016;17:99-105.

13. Lu L, Yi Y. Acupuncture and moxibustion for treatment 
of 21 cases of restless legs syndrome. World J Acu Moxi 2006;16:63-4.

14. Dai X, Li Y, Song Q, et al. Clinical observation of warm acupuncture at treating post-apoplectic restless legs syndrome Biguan (ST 31). J Acupunct Tuina Sci 2006; 4:174.

15. Di Stanislao C, Brotzu R, Franconi G. Acupuncture for restless legs syndrome: A retrospective case series. Med Acupunt 2009;21:63-5.

16. Jiang J. Acupuncture treatment of restless leg syndrome in 31 cases. Zhejiang J Traditional Chin Med 2013;48:352.

17. Li L, Wang G, Wen F, et al. Therapeutic observation of acupuncture on restless legs syndrome. Chin Arch Traditional Chin Med 2007;25:621-2.

18. Li Y, Chen W. Acupuncture treatment of restless legs syndrome. J Practical Traditional Chin Med 2011;12:848-9.

19. Liu G. Treatment of 49 cases of restless legs syndrome by acupuncture plus acupoint injection. J Acpunct Tuina Sci 2006;4:50-1.

20. Liu J, Zou W, Liu Y. Observation on the therapeutic effect of acupuncture on the treatment of restless leg syndrome. JCAM 2011;27:25-6.

21. Pan W, Wang M, Li M, et al. Actigraph evaluation of acupuncture for treating restless legs syndrome. Evid Based Complement Alternat Med 2015;2015:343201.

22. Raissi GR, Forogh B, Ahadi T, et al. Evaluation of Acupuncture in the Treatment of Restless Legs Syndrome: A Randomized Controlled Trial. J Acupunct Meridian Stud 2017;10:346-50.

23. Shi L, Wang Y. Clinical observation on 40 cases of restless leg syndrome treated with acupuncture and Chinese herbal fumigation. Chin Acupunct Moxibustion 2003;23:651-2.

24. Tang S, Tang P. Effect of acupuncture combined with drugs on sleep quality in patients with restless legs syndrome. Liaoning J Traditional Chin Med 2009;36:114-5.

25. Tian Z. Acupuncture treatment of 76 cases of restless leg syndrome. Chinese Acupunct Moxibustion 1994;S1:45.

26. Wang J, Zhang J. Observation on the therapeutic effect of acupuncture combined with walking cans for the treatment of restless legs syndrome. Shanghai J Acu-mox 2011;30:836-7.

27. Wu YH, Sun CL, Wu D, et al. Observation on therapeutic effect of acupuncture on restless legs syndrome. Zhongguo Zhen Jiu 2008;28:27-9.

28. Yu J, Sun Z, Li H, et al. Clinical observation of acupuncture treatment of restless legs syndrome. China
Med 2016;11:1397-8.

29. Zhang Y, Xue Z, Wang L. Therapeutic effect of acupuncture and spleen and stomach on primary restless leg syndrome. Shaanxi Traditional Chin Med 2014;35:1069-71.

30. Zhou W, Wang DH, Yang RK, et al. Comparison of therapeutic effects of acupoints selected along different meridians on restless legs syndrome. Zhongguo Zhen Jiu 2005;25:616-8.

31. Xu XM, Liu Y, Jia SY, et al. Complementary and alternative therapies for restless legs syndrome: An evidence-based systematic review. Sleep Med Rev 2018;38:158-67.

32. Zeng BY, Salvage S, Jenner P. Current development of acupuncture research in Parkinson's disease. Int Rev Neurobiol 2013;111:141-58.

33. Xiao D. Acupuncture for Parkinson's Disease: a review of clinical, animal, and functional Magnetic Resonance Imaging studies. J Tradit Chin Med 2015;35:709-17.

34. Cripps MG. Acupuncture for restless legs syndrome in patients previously treated with dopaminergic drugs. Acupunct Med 2011;29:240-1.

35. Mohammadi MM, Raygani AAV, Ghobadi A, et al. Effect of Near-Infrared Light Therapy Based on Acupoints on the Severity of Restless Legs Syndrome in Patients Undergoing Hemodialysis: A Single-Blind, Randomized Controlled Trial. Clin Med Res 2018;16:1-8.

36. Fukutome T. Acupuncture point injection markedly improved sensory symptoms and motor signs in 2 patients with restless legs syndrome. Clin Case Rep 2018;6:1353-7.

37. Harrison EG, Keating JL, Morgan PE. Nonpharmacological interventions for restless legs syndrome: a systematic review of randomised controlled trials. Disabil Rehabil 2019;41:2006-14.

38. Szentkiralyi A, Fendrich K, Hoffmann W, et al. Socioeconomic risk factors for incident restless legs syndrome in the general population. J Sleep Res 2012;21:561-8.

39. Koo BB, Blackwell T, Lee HB, et al. Restless Legs Syndrome and Depression: Effect Mediation by Disturbed Sleep and Periodic Limb Movements. Am J Geriatr Psychiatry 2016;24:1105-16.

40. Page RL 2nd, Ruscin JM, Bainbridge JL, et al. Restless legs syndrome induced by escitalopram: case report and review of the literature. Pharmacotherapy 2008;28:271-80.

41. Metheny J. Acupuncture and the placebo effect. Arch Ophthalmol 2011;129:1107-8; author reply 1108.

42. Slack R. 2010. Acupuncture: a science-based assessment: a position paper from the Center for Inquiry Office 
of Public Policy. Center for Inquiry. Available online: http://www.centerforinquiry.net /uploads/attachments/ Acupuncture_Paper.pdf

43. Barth J, Muff S, Kern A, et al. Effect of Briefing on Acupuncture Treatment Outcome Expectations, Pain, and Adverse Side Effects Among Patients With Chronic Low
Back Pain: A Randomized Clinical Trial. JAMA Netw Open 2021;4:e2121418.

44. Metheny J. Acupuncture and the placebo effect. Arch Ophthalmol 2011;129:1107-8; author reply 1108.

(English Language Editor: J. Jones)

Cite this article as: Huang C, Tang JF, Sun W, Wang LZ, Jin YS. Effectiveness of acupuncture in the management of restless leg syndrome: a systematic review and meta-analysis. Ann Palliat Med 2021;10(10):10495-10505. doi: 10.21037/apm-212309 